\title{
YAMABE FLOW WITH PRESCRIBED SCALAR CURVATURE
}

\author{
INAS AMACHA AND RACHID REGBAOUI
}

\begin{abstract}
In this work, we study the Yamabe flow corresponding to the prescribed scalar curvature problem on compact Riemannian manifolds with negative scalar curvature. The long time existence and convergence of the flow are proved under appropriate conditions on the prescribed scalar curvature function.
\end{abstract}

\section{INTRODUCTION}

The prescribed scalar curvature problem on a compact Riemannian manifold $\left(M, g_{0}\right)$ of dimension $n \geq 3$, consists of finding a conformal metric $g$ to $g_{0}$ whose scalar curvature $R_{g}$ is equal to a given function $f \in C^{\infty}(M)$. If we set $g=u^{\frac{4}{n-2}} g_{0}$, where $0<u \in C^{\infty}(M)$, then we have

$$
R_{g}=u^{-\frac{n+2}{n-2}}\left(-c_{n} \Delta u+R_{0} u\right)
$$

where $\Delta$ is the Laplace operator associated with $g_{0}, R_{0}$ is the scalar curvature of $g_{0}$ and $c_{n}=4 \frac{n-1}{n-2}$.

Then the prescribed scalar curvature problem :

$$
R_{g}=f
$$

is equivalent to solve the following nonlinear PDE :

$$
-c_{n} \Delta u+R_{0} u=f u^{\frac{n+2}{n-2}}
$$

on the space of smooth positive functions on $M$. The solvability of this equation depends on $R_{0}$ and the prescribed function $f$. When $f$ is constant, equation (1.1) becomes the famous Yamabe equation whose resolution has been for a long time a challenging problem in geometric Analysis. See [2], 12, 14], 16], 17], for more details on the Yamabe problem, and 1], [5], 10, 13], [15, 21], concerning the prescribed scalar curvature problem.

By changing conformally $g_{0}$ if necessary, we may always assume that $R_{0}$ satisfies one of the conditions : $R_{0}>0, R_{0}=0$ or $R_{0}<0$ everywhere on $M$. Equation (1.1) has a variational structure since there are different functionals whose Euler-Lagrange equations are equivalent to (1.1). When $R_{0}<0$, the following functional seems more appropriate to handle the prescribed scalar curvature problem :

$$
\mathcal{E}(g)=\int_{M} R_{g} d V_{g}-\frac{n-2}{n} \int_{M} f d V_{g}
$$

2000 Mathematics Subject Classification. 35K55, 58J35, 53A30.

Key words and phrases. conformal metric, scalar curvature, Yamabe flow. 
where $g=u^{\frac{4}{n-2}} g_{0}$ belongs to the conformal class $\left[g_{0}\right]$ of $g_{0}, R_{g}$ is the scalar curvature of $g$ and $d V_{g}=u^{\frac{2 n}{n-2}} d V_{g_{0}}$ is the volume element of $g$.

Simple computations (44) show that the $L^{2}$-gradient of $\mathcal{E}$ is $\frac{n-2}{2 n}\left(R_{g}-f\right) g$, and then, after changing time by a constant scale, the associated negative gradient flow equation is

$$
\left\{\begin{array}{l}
\partial_{t} g=-\left(R_{g}-f\right) g \\
g(0)=g^{0}
\end{array}\right.
$$

where $g^{0}=u_{0}^{\frac{4}{n-2}} g_{0}$ is a given metric in the conformal class of $g_{0}$.

Since equation (1.3) preserves the conformal structure of $M$, then any smooth solution of (1.3) is of the form $g(t)=u(t)^{\frac{4}{n-2}} g_{0}$, where $0<u(t) \in C^{\infty}(M)$. For simplicity we have used the notation $u(t):=u(t,),. t \in I$ for any function $u$ defined on $I \times M$, where $I$ is a subset of $\mathbb{R}$. In terms of $u(t)$, the flow (1.3) may be written in the equivalent form :

$$
\left\{\begin{array}{l}
\partial_{t} u^{N}=\frac{n+2}{4}\left(c_{n} \Delta u-R_{0} u+f u^{N}\right) \\
u(0)=u_{0} \in C^{\infty}(M), u_{0}>0
\end{array}\right.
$$

where $N=\frac{n+2}{n-2}$.

Our aim in this paper is to investigate this gradient flow by proving its longtime existence and analysing its asymptotic behavior when $t \rightarrow+\infty$.

Our first result is the following existence theorem :

Theorem 1.1. Suppose that $R_{0}<0$ and let $f \in C^{\infty}(M)$. Then for any $g^{0}=u_{0}^{\frac{4}{n-2}} g_{0}$ with $0<u_{0} \in C^{\infty}(M)$, there exists a unique solution $g(t)=u(t)^{\frac{4}{n-2}} g_{0}$ of $(1.3)$ defined on $[0,+\infty)$, where $0<u \in C^{\infty}([0,+\infty) \times M)$. Moreover, the functional $\mathcal{E}$ is decreasing along the solution $g(t)$, that's

$$
\frac{d}{d t} \mathcal{E}(g(t)) \leq 0 \text { for all } t \in[0,+\infty)
$$

We note here that apart from the smoothness of $f$, no further assumptions on the function $f$ are needed in Theorem 1.1. However, for the longtime behavior, it is necessary to assume additional condition in order to get the convergence of the flow. Indeed, if $f \geq 0$, by applying the maximum principe to (1.4), one can easily check that

$$
u(t) \geq\left(\min _{M}\left|R_{0}\right| \min _{M} u_{0}\right) t^{\frac{n-2}{4}} \rightarrow+\infty \text { as } t \rightarrow+\infty .
$$

So if one wants to get the convergence of the flow, it is necessary to assume at least that $f$ is negative somewhere on $M$. We note that this last condition is also necessary to the resolution of equation (1.1) since it is well known that it if the negative gradient flow associated with a functional $\mathcal{F}$ converges (in some sense), then its limit is a critical point of $\mathcal{F}$. 
Before giving conditions on $f$ ensuring the convergence of the flow, let us fix some notations: if $\Omega \subset M$ is an open set, we denote by $\lambda_{\Omega}$ the first eigenvalue of the conformal Laplacian $L=$ $-c_{n} \Delta+R_{0}$ on $\Omega$ with zero Dirichlet boundary conditions, that is

$$
\lambda_{\Omega}=\inf _{0 \neq u \in H_{0}^{1}(\Omega)} \frac{\int_{M}\left(c_{n}|\nabla u|^{2}+R_{0} u^{2}\right) d V_{g_{0}}}{\int_{M} u^{2} d V_{g_{0}}} .
$$

We then assume the following conditions on $f$ :

There exists an open set $\Omega \subset M$ such that

$$
\lambda_{\Omega}>0 \text { and } f<0 \text { on } M \backslash \Omega
$$

and

$$
\sup _{x \in \Omega} f(x) \leq C_{\Omega} \inf _{x \in M \backslash \Omega}|f(x)|,
$$

where $C_{\Omega}$ is a positive constant depending only on $\Omega$.

We then have the following result :

Theorem 1.2. Suppose that $R_{0}<0$ and that $f \in C^{\infty}(M)$ satisfies conditions $(H 1)-(H 2)$. Then there exists a function $0<\bar{u} \in C^{\infty}(M)$ such that for any smooth metric $g^{0}=u_{0}^{\frac{4}{n-2}} g_{0}$ with $0<u_{0} \leq \bar{u}$, the flow $g(t)=u(t)^{\frac{4}{n-2}} g_{0}$ given by Theorem 1.1 converges in the $C^{\infty}$-topology to a conformal metric $g_{\infty}=u_{\infty}^{\frac{4}{n-2}} g_{0}$ whose scalar curvature is $f$, that's, $R_{g_{\infty}}=f$.

A particular interesting case is when the function $f$ satisfies $f(x)<0$ for almost all $x \in M$. In this case conditions (H1)-(H2) are automatically satisfied and then we have the following corollary:

Corollary 1.1. Suppose that $R_{0}<0$ and $f \in C^{\infty}(M)$ such that $f<0$ almost everywhere on $M$.

Then there exists a function $0<\bar{u} \in C^{\infty}(M)$ such that for any smooth metric $g^{0}=u_{0}^{\frac{4}{n-2}} g_{0}$ with $0<u_{0} \leq \bar{u}$, the flow $g(t)=u(t)^{\frac{4}{n-2}} g_{0}$ given by Theorem 1.1 converges in the $C^{\infty}$-topology to a conformal metric $g_{\infty}=u_{\infty}^{\frac{4}{n-2}} g_{0}$ whose scalar curvature is $f$, that's, $R_{g_{\infty}}=f$.

It is naturel to ask if conditions (H1)-(H2) in Theorem 1.2 are necessary. The following Theorem tells us that this is the case at least for condition (H1).

Theorem 1.3. Suppose that $R_{0}<0$ and let $f \in C^{\infty}(M)$ such that condition (H1) is not satisfied, that's, for any open set $\Omega \subset M$ such that $f>0$ on $M \backslash \Omega$, we suppose $\lambda_{\Omega} \leq 0$. Then for any $0<u_{0} \in C^{\infty}(M)$, the solution $u(t)$ of (1.4) satisfies for some constant $C>0$ depending only on $u_{0}, g_{0}, f$,

$$
\max _{x \in M} u(t, x) \geq C t^{\frac{n-2}{n+2}} \rightarrow+\infty \text { as } t \rightarrow+\infty
$$


We note here that condition (H1) is conformally invariant. Similar conditions to (H1)-(H2) were found by many authors to solve (1.1) by the direct method of elliptic PDEs, see ([5], [15, [21]) for more details. To our knowledge, the only known results on Yamabe type flow on dimension $n \geq 3$ concern the case where $f$ is constant or $M=\mathbb{S}^{n}$. The Yamabe flow was first introduced by Hamilton [11] and has been the subject of several studies, see [6, [7, [8], [18, [22. When $f$ is non constant, we mention the work of Struwe [20] about the Nirenberg's problem on the sphere $\mathbb{S}^{2}$, and the results of Chen-Xu [9] concerning $\mathbb{S}^{n}, n \geq 3$. A general evolution problem related to the prescribed Gauss curvature on surfaces was studied by Baird-Fardoun-Regbaoui [3].

The paper is organized as follows. In section 2 we prove the global existence of the flow by establishing local $C^{k}$-estimates on the solution $u$ of (1.4). In section 3, we study the asymptotic behavior of the flow when $t \rightarrow+\infty$. In particular we prove uniform $C^{k}$-estimates on $u$ which are necessary to get the convergence of the flow.

\section{GLOBAL EXISTENCE OF THE FLOW}

In this section we shall establish some estimates on the solution $u$ of (1.4) which will be an important tool in proving that the flow $g(t)$ is globally defined on $[0,+\infty)$. In this section we suppose that $R_{0}<0$ and $f \in C^{\infty}(M)$.

As already mentioned in the previous section, equation (1.3) is equivalent to (1.4), so it suffices to prove the existence of a solution $u(t)$ of (1.4) defined on $[0,+\infty)$ to obtain a metric $g(t)$ solution of (1.3) defined on $[0,+\infty)$. Since (1.4) is a parabolic equation (on the set of smooth positive functions on $[0, T) \times M$, for any $T>0)$, then there exists a smooth solution $u(t)$ of (1.4) defined on a maximal interval $\left[0, T^{*}\right)$ satisfying $u(t)>0$ on $\left[0, T^{*}\right)$. Thus we have a solution $g(t)=u^{\frac{4}{n-2}} g_{0}$ of (1.3) defined on a maximal interval $\left[0, T^{*}\right)$. For simplicity, we shall write $u$ instead of $u(t)$ and $g$ instead of $g(t)$.

Now, we derive some properties on $g$ which will be important later. One can check by using (1.4) that the scalar curvature $R_{g}$ satisfies the following equation

$$
\partial_{t} R_{g}=(n-1) \Delta_{g}\left(R_{g}-f\right)+R_{g}\left(R_{g}-f\right)
$$

where $\Delta_{g}$ is the Laplacian associated with $g(t)$.

A simple computation using (2.1) gives

$$
\frac{d}{d t} \mathcal{E}(g)=-\frac{n-2}{2} \int_{M}\left(R_{g}-f\right)^{2} d V_{g}
$$

so the functional $\mathcal{E}$ is decreasing along the flow $g(t)$. If we set

$$
E(u):=\mathcal{E}(g)=\mathcal{E}\left(u^{\frac{4}{n-2}} g_{0}\right)=\int_{M}\left(c_{n}|\nabla u|^{2}+R_{0} u^{2}-\frac{n-2}{n} f u^{\frac{2 n}{n-2}}\right) d V_{g_{0}},
$$

then $(2.2)$ can be written in terms of $u$ :

$$
\frac{d}{d t} E(u)=-\frac{8}{n-2} \int_{M}\left|\partial_{t} u\right|^{2} u^{\frac{4}{n-2}} d V_{g_{0}} \leq 0
$$


The following lemma will be very useful to prove integral estimates on the solution $g$.

Lemma 2.1. We have for any $p>1$,

$$
\begin{aligned}
& \frac{d}{d t} \int_{M}\left|R_{g}-f\right|^{p} d V_{g}=-\frac{4(n-1)(p-1)}{p} \int_{M}\left|\nabla_{g}\right| R_{g}-\left.\left.f\right|^{\frac{p}{2}}\right|_{g} ^{2} d V_{g} \\
& +\left(p-\frac{n}{2}\right) \int_{M}\left(R_{g}-f\right)\left|R_{g}-f\right|^{p} d V_{g}+p \int_{M} f\left|R_{g}-f\right|^{p} d V_{g},
\end{aligned}
$$

where $\nabla_{g}$ is the gradient with respect to the metric $g$ and $\left.||\right|_{g$.$} is the Riemannian norm with respect$ to $g$.

Proof. We have for any $p \geq 1$

$$
\frac{d}{d t} \int_{M}\left|R_{g}-f\right|^{p} d V_{g}=p \int_{M}\left|R_{g}-f\right|^{p-2}\left(R_{g}-f\right) \partial_{t} R_{g} d V_{g}+\frac{1}{2} \int_{M} R_{g} t r_{g}\left(\partial_{t} g\right) d V_{g} .
$$

Using equations (1.3) and (2.1) it follows that

$$
\begin{gathered}
\frac{d}{d t} \int_{M}\left|R_{g}-f\right|^{p} d V_{g}=(n-1) p \int_{M}\left|R_{g}-f\right|^{p-2}\left(R_{g}-f\right) \Delta_{g}\left(R_{g}-f\right) \\
+p \int_{M} R_{g}\left|R_{g}-f\right|^{p}-\frac{n}{2} \int_{M}\left|R_{g}-f\right|^{p}\left(R_{g}-f\right) d V_{g} \\
=-\frac{4(n-1)(p-1)}{p} \int_{M}\left|\nabla_{g}\right| R_{g}-\left.\left.f\right|^{\frac{p}{2}}\right|_{g} ^{2} d V_{g}+\left(p-\frac{n}{2}\right) \int_{M}\left(R_{g}-f\right)\left|R_{g}-f\right|^{p} d V_{g} \\
+p \int_{M} f\left|R_{g}-f\right|^{p} d V_{g} .
\end{gathered}
$$

In order to prove that the solution $g(t)=u(t)^{\frac{4}{n-2}} g_{0}$ is globally defined on $[0,+\infty)$, we need upper and lower bounds on $u(t)$.

Proposition 2.1. Let $g(t)=u(t)^{\frac{4}{n-2}} g_{0}$ be the solution of (1.3) defined on a maximal interval $\left[0, T^{*}\right)$. Then we have for any $t \in\left[0, T^{*}\right)$ :

$$
\min \left(C_{0}, \min _{M} u_{0}\right) \leq u(t) \leq \max \left(1, \max _{M} u_{0}\right) e^{C_{1} t},
$$

where $C_{0}=\left(\frac{\min _{M}\left|R_{0}\right|}{\max _{M}|f|}\right)^{\frac{n-2}{4}}, C_{1}=\frac{n-2}{4}\left(\max _{M}\left|R_{0}\right|+\max _{M}|f|\right)$.

Proof. The proof uses an elementary maximum principal argument. Indeed, fix $t \in[0, T)$ and let $\left(t_{0}, x_{0}\right) \in[0, t] \times M$ such that $u\left(t_{0}, x_{0}\right)=\min _{[0, t] \times M} u$. If $t_{0}=0$, then

$$
\min _{[0, t] \times M} u=\min _{M} u_{0}
$$

so the first inequality in (2.4) is proved in this case. Now suppose that $t_{0}>0$. We have then $\partial_{t} u\left(t_{0}, x_{0}\right) \leq 0$ and $\Delta u\left(t_{0}, x_{0}\right) \geq 0$. Thus we obtain after substituting in (1.4) that

$$
0 \geq-R_{0}\left(x_{0}\right) u\left(t_{0}, x_{0}\right)+f\left(x_{0}\right) u^{N}\left(t_{0}, x_{0}\right)
$$


which implies that

$$
u\left(t_{0}, x_{0}\right) \geq\left(\frac{\min _{M}\left|R_{0}\right|}{\max _{M}|f|}\right)^{\frac{1}{N-1}},
$$

where $N=\frac{n+2}{n-2}$. This proves the first inequality in (2.4). In order to prove the second inequality we set $v=e^{-C_{1} t} u$ instead of $u$, where $C_{1}=\frac{4}{n-2}\left(\max _{M}\left|R_{0}\right|+\max _{M}|f|\right)$. As above, fix $t \in[0, T)$ and let $\left(t_{0}, x_{0}\right) \in[0, t] \times M$ such that $v\left(t_{0}, x_{0}\right)=\max _{[0, t] \times M} v$. If $t_{0}=0$, then $\max _{[0, t] \times M} v=\max _{M} u_{0}$, which implies

$$
\max _{[0, t] \times M} u \leq \max _{M} u_{0} e^{C_{1} t}
$$

so the second inequality in (2.4) is proved in this case. Now suppose that $t_{0}>0$. We have then $\partial_{t} v\left(t_{0}, x_{0}\right) \geq 0$ and $\Delta v\left(t_{0}, x_{0}\right) \leq 0$, that's, $\partial_{t} u\left(t_{0}, x_{0}\right) \geq C_{1} u\left(t_{0}, x_{0}\right)$ and $\Delta u\left(t_{0}, x_{0}\right) \leq 0$. We obtain after substituting in (1.4) that

$$
N C_{1} u^{N}\left(t_{0}, x_{0}\right) \leq \frac{n+2}{4}\left(-R_{0}\left(x_{0}\right) u\left(t_{0}, x_{0}\right)+f\left(x_{0}\right) u^{N}\left(t_{0}, x_{0}\right)\right)
$$

which implies that

$$
u\left(t_{0}, x_{0}\right) \leq 1
$$

since $N C_{1}=\frac{n+2}{4}\left(\max _{M}\left|R_{0}\right|+\max _{M}|f|\right)$. It is clear that (2.5) implies that

$$
\max _{[0, t] \times M} u \leq e^{C_{1} t}
$$

The Proof of Proposition 2.1 is then complete.

Now we prove integral estimates on $R_{g}$ which will imply estimates on $\partial_{t} u$ :

Proposition 2.2. Let $g(t)$ be the solution of (1.3) defined on a maximal interval $\left[0, T^{*}\right)$. Then we have for any $t \in\left[0, T^{*}\right)$,

$$
\int_{M}\left|R_{g(t)}-f\right|^{p} d V_{g(t)} \leq C e^{C t}
$$

where $p=\frac{n^{2}}{2(n-2)}$ and $C$ is a positive constant depending only on $f, g_{0}, u_{0}$.

Proof. In what follows $C$ denotes a positive constant depending on $f, g_{0}, u_{0}$, whose value may change from line to line.

We have by Lemma 2.1 for any $t \in\left[0, T^{*}\right)$

$$
\begin{aligned}
& \frac{d}{d t} \int_{M}\left|R_{g}-f\right|^{p} d V_{g}=-\frac{4(n-1)(p-1)}{p} \int_{M}\left|\nabla_{g}\right| R_{g}-\left.\left.f\right|^{\frac{p}{2}}\right|_{g} ^{2} d V_{g} \\
& +\left(p-\frac{n}{2}\right) \int_{M}\left(R_{g}-f\right)\left|R_{g}-f\right|^{p} d V_{g}+p \int_{M} f\left|R_{g}-f\right|^{p} d V_{g}
\end{aligned}
$$

where $\nabla_{g}$ is the gradient with respect to the metric $g$ and $|\cdot|_{g}$ is the Riemannian norm with respect to $g$. 


$$
\begin{aligned}
& \frac{d}{d t} \int_{M}\left|R_{g}-f\right|^{p} d V_{g}+\frac{4(n-1)(p-1)}{p} \int_{M}\left|\nabla_{g}\right| R_{g}-\left.\left.f\right|^{\frac{p}{2}}\right|_{g} ^{2} d V_{g} \\
& \quad \leq\left|p-\frac{n}{2}\right| \int_{M}\left|R_{g}-f\right|^{p+1} d V_{g}+C \int_{M}\left|R_{g}-f\right|^{p} d V_{g} .
\end{aligned}
$$

By (2.4) we have

and

$$
\int_{M}\left|\nabla_{g}\right| R_{g}-\left.\left.f\right|^{\frac{p}{2}}\right|_{g} ^{2} d V_{g}=\int_{M}|\nabla| R_{g}-\left.\left.f\right|^{\frac{p}{2}}\right|^{2} u^{2} d V_{g_{0}} \geq C \int_{M}|\nabla| R_{g}-\left.\left.f\right|^{\frac{p}{2}}\right|^{2} d V_{g_{0}}
$$

$$
\int_{M}\left|R_{g}-f\right|^{p} d V_{g}=\int_{M}\left|R_{g}-f\right|^{p} u^{\frac{2 n}{n-2}} d V_{g_{0}} \geq C \int_{M}\left|R_{g}-f\right|^{p} d V_{g_{0}} .
$$

By Sobolev's inequality we have

$$
\left(\int_{M}\left|R_{g}-f\right|^{\frac{p n}{n-2}} d V_{g_{0}}\right)^{\frac{n-2}{n}} \leq C\left(\int_{M}|\nabla| R_{g}-\left.\left.f\right|^{\frac{p}{2}}\right|^{2} d V_{g_{0}}+\int_{M}\left|R_{g}-f\right|^{p} d V_{g_{0}}\right)
$$

which gives by using (2.4) that

$$
\left(\int_{M}\left|R_{g}-f\right|^{\frac{p n}{n-2}} d V_{g}\right)^{\frac{n-2}{n}} \leq C e^{C t}\left(\int_{M}|\nabla| R_{g}-\left.\left.f\right|^{\frac{p}{2}}\right|^{2} d V_{g_{0}}+\int_{M}\left|R_{g}-f\right|^{p} d V_{g_{0}}\right) .
$$

It follows from $(2.8),(2.9),(2.10)$ and (2.11) that

$$
\begin{aligned}
& \frac{d}{d t} \int_{M}\left|R_{g}-f\right|^{p} d V_{g}+C^{-1} e^{-C t}\left(\int_{M}\left|R_{g}-f\right|^{\frac{p n}{n-2}} d V_{g}\right)^{\frac{n-2}{n}} \\
& \quad \leq\left(p-\frac{n}{2}\right) \int_{M}\left|R_{g}-f\right|^{p+1} d V_{g}+C \int_{M}\left|R_{g}-f\right|^{p} d V_{g}
\end{aligned}
$$

By taking $p=\frac{n}{2}$ in (2.12) we get

which implies that

$$
\frac{d}{d t} \int_{M}\left|R_{g}-f\right|^{p} d V_{g} \leq C \int_{M}\left|R_{g}-f\right|^{p} d V_{g}
$$

$$
\int_{M}\left|R_{g}-f\right|^{\frac{n}{2}} d V_{g} \leq C e^{C t} .
$$

Now taking again $p=\frac{n}{2}$ in (2.12) and integrating on $[0, t], t \in\left[0, T^{*}\right)$, we obtain by using $(2.13)$

$$
\int_{0}^{t}\left(\int_{M}\left|R_{g(s)}-f\right|^{\frac{n^{2}}{2(n-2)}} d V_{g(s)}\right)^{\frac{n-2}{n}} d s \leq C e^{C t} .
$$

We have by Hölder's inequality and Young's inequality, for any $\varepsilon>0$ and $p>\frac{n}{2}$,

$$
\int_{M}\left|R_{g}-f\right|^{p+1} d V_{g} \leq \varepsilon\left(\int_{M}\left|R_{g}-f\right|^{\frac{p n}{n-2}} d V_{g}\right)^{\frac{n-2}{n}}+\varepsilon^{-\frac{n}{2 p-n}}\left(\int_{M}\left|R_{g}-f\right|^{p} d V_{g}\right)^{\frac{2 p-n+2}{2 p-n}}
$$


If we combine (2.15) with (2.12) and taking $\varepsilon=\left(p-\frac{n}{2}\right)^{-1} C^{-1} e^{-C t}$, we get

that's

$$
\frac{d}{d t} \int_{M}\left|R_{g}-f\right|^{p} d V_{g} \leq C e^{C t}\left(\int_{M}\left|R_{g}-f\right|^{p} d V_{g}\right)^{\frac{2 p-n+2}{2 p-n}}+C \int_{M}\left|R_{g}-f\right|^{p} d V_{g}
$$

$$
\frac{d}{d t} \log \left(\int_{M}\left|R_{g}-f\right|^{p} d V_{g}\right) \leq C\left(e^{C t}\left(\int_{M}\left|R_{g}-f\right|^{p} d V_{g}\right)^{\frac{2}{2 p-n}}+1\right)
$$

In particular by choosing $p=\frac{n^{2}}{2(n-2)}$ and integrating on $[0, t], t \in\left[0, T^{*}\right)$, we obtain

$$
\begin{gathered}
\log \left(\int_{M}\left|R_{g(t)}-f\right|^{\frac{n^{2}}{2(n-2)}} d V_{g(t)}\right) \leq \log \left(\int_{M}\left|R_{g(0)}-f\right|^{\frac{n^{2}}{2(n-2)}} d V_{g(0)}\right) \\
+C e^{C t} \int_{0}^{t}\left(\int_{M}\left|R_{g(s)}-f\right|^{\frac{n^{2}}{2(n-2)}} d V_{g(s)}\right)^{\frac{n-2}{n}} d s+C t
\end{gathered}
$$

which by using (2.14) gives

This proves Proposition 2.2.

$$
\log \left(\int_{M}\left|R_{g(t)}-f\right|^{\frac{n^{2}}{2(n-2)}} d V_{g(t)}\right) \leq C e^{t}
$$

With the estimates of Proposition 2.1 one would like to apply the classical Shauder estimates for parabolic equations. To this end we need $C^{\alpha}$-estimates :

Proposition 2.3. Let $g(t)=u(t)^{\frac{4}{n-2}} g_{0}$ be the solution of (1.3) defined on a maximal interval $\left[0, T^{*}\right)$. Then we have for some $\alpha \in(0,1)$ and any $T \in\left[0, T^{*}\right)$

$$
\|u\|_{C^{\alpha}([0, T] \times M)} \leq C e^{C T}
$$

where $C$ is a positive constant depending only on $u_{0}, g_{0}$ and $f$.

Proof. By using Proposition 2.1 and Proposition 2.2, the proof is identical to that of Proposition 2.6 in Brendle [6].

Proof of Theorem 1.1. Let $g(t)=u(t)^{\frac{4}{n-2}} g_{0}$ be the solution of (1.3) defined on a maximal interval $\left[0, T^{*}\right)$. Assume by contradiction that $T^{*}<+\infty$. Then by using Proposition 2.1 and Proposition 2.3 we have

$$
\|u\|_{C^{\alpha}\left(\left[0, T^{*}\right) \times M\right)} \leq C e^{C T^{*}} \text { and } \min _{\left[0, T^{*}\right) \times M} u \geq \min \left(C_{0}, \min _{M} u_{0}\right) .
$$

for some $\alpha \in(0,1)$, where $C$ is a positive constant depending $u_{0}, f, g_{0}$. The classical theory of linear parabolic equations applied to (1.4) implies that $u$ is bounded in $C^{k}\left(\left[0, T^{*}\right) \times M\right)$ for any $k \in \mathbb{N}$, that's

$$
\|u\|_{C^{k}\left(\left[0, T^{*}\right) \times M\right)} \leq C_{k},
$$

where $C_{k}$ is a positive constant depending only on $u_{0}, g_{0}, f$ and $k$. It is clear that (2.16) allows us to extend the solution beyond $T^{*}$ contradicting thus the maximality of $T^{*}$. We see from (2.2) that the functional $\mathcal{E}$ is decreasing along the flow. The proof of Theorem 1.1 is then complete. 


\section{Long Time BeHAVIOR OF THE FLOW}

In this section we study the asymptotic behavior of the flow $g(t)$ when $t \rightarrow+\infty$. First we prove the following proposition which gives a super solution of equation (1.1) when conditions (H1)-(H2) are satisfied.

Proposition 3.1. Suppose that there exists an open set $\Omega \subset M$ such that conditions $(H 1)-(H 2)$ are satisfied. Then there exists a conformal metric $\bar{g}=\bar{u}^{\frac{4}{n-2}} g_{0}, 0<\bar{u} \in C^{\infty}(M)$, satisfying

or equivalently

$$
R_{\bar{g}}-f \geq 0
$$

$$
-c_{n} \Delta \bar{u}+R_{0} \bar{u}-f \bar{u}^{N} \geq 0, N=\frac{n+2}{n-2} .
$$

Proof. By hypothesis, there is an open set $\Omega \subset M$ satisfying $(H 1)-(H 2)$, that's

$$
\lambda_{\Omega}>0 \text { and } f<0 \text { on } M \backslash \Omega
$$

and

$$
\sup _{x \in \Omega} f(x) \leq C_{\Omega} \inf _{x \in M \backslash \Omega}|f(x)|,
$$

where $C_{\Omega}$ is a positive constant depending only on $\Omega$.

Let $\varepsilon>0$ and set

$$
\Omega_{\varepsilon}=\{x \in M: d(x, \Omega)<\varepsilon\} .
$$

For $\varepsilon>0$ sufficiently small we have from (H1) that $\lambda_{\Omega_{\varepsilon}}>0$, where $\lambda_{\Omega_{\varepsilon}}$ is the first eigenvalue of the operator $-c_{n} \Delta+R_{0}$ on $\Omega_{\varepsilon}$ with zero Dirichlet boundary conditions. Let $D \subset M$ be an open set of smooth boundary such that $\bar{\Omega} \subset D \subset \Omega_{\varepsilon}$. Then we have $\lambda_{D} \geq \lambda_{\Omega_{\varepsilon}}>0$. Let $\varphi_{0}$ an eigenfunction associated with $\lambda_{D}$, that's

$$
-c_{n} \Delta \varphi_{0}+R_{0} \varphi_{0}=\lambda_{D} \varphi_{0}
$$

Then we have that $\varphi_{0} \in C^{\infty}(\bar{D})$ and using the maximum principle of elliptic equations one has $\varphi_{0}>0$ on $D$. By normalising if necessary, we may suppose that

$$
0<\varphi_{0} \leq 1 \text { on } D
$$

Let $\chi \in C_{0}^{\infty}(D)$ such that $0 \leq \chi \leq 1$ and $\chi=1$ on $\bar{\Omega}$. We define the function $\bar{u} \in C^{\infty}(M)$ by setting

$$
\bar{u}=\delta\left(\chi \varphi_{0}+1-\chi\right),
$$

where $\delta>0$ will be chosen later. By (3.3) and the definition of $\chi$ it is easy to check that 


$$
m_{0}:=\inf _{M}\left(\chi \varphi_{0}+1-\chi\right)>0
$$

so

$$
\bar{u} \geq \delta m_{0} .
$$

Now let us prove that $\bar{u}$ satisfies (3.2). If we set

$$
\mathcal{L}(\bar{u})=-c_{n} \Delta \bar{u}+R_{0} \bar{u}-f \bar{u}^{\frac{n+2}{n-2}},
$$

then (3.2) is equivalent to $\mathcal{L}(\bar{u}) \geq 0$.

A simple computation shows that we have on $\Omega$ (using the fact that $\chi=1$ on $\bar{\Omega}$ ):

$$
\mathcal{L}(\bar{u})=\lambda_{D} \delta \varphi_{0}-f \delta^{N} \varphi_{0}^{N}=\delta \varphi_{0}\left(\lambda_{D}-\delta^{N-1} f \varphi_{0}^{N-1}\right)
$$

and by using (3.3) it follows that

$$
\mathcal{L}(\bar{u}) \geq \delta \varphi_{0}\left(\lambda_{D}-\delta^{N-1} \sup _{x \in \Omega} f(x)\right)
$$

It follows from (3.5) that if we want $\mathcal{L}(\bar{u}) \geq 0$ on $\Omega$, we have to choose $\delta>0$ satisfying

$$
\delta^{N-1} \sup _{x \in \Omega} f(x) \leq \lambda_{D}
$$

Now we examine the sign of $\mathcal{L}(\bar{u})$ on $M \backslash \Omega$. We have from the definition of $\bar{u}$ that

$$
\mathcal{L}(\bar{u})=\delta\left(-c_{n} \Delta+R_{0}\right)\left(\chi \varphi_{0}+1-\chi\right)-f \bar{u}^{N} .
$$

By using (3.4) and the fact that $f<0$ on $M \backslash \Omega$, it follows from (3.7)

$$
\mathcal{L}(\bar{u}) \geq-\delta m_{1}+\delta^{N} m_{0}^{N} \inf _{x \in M \backslash \Omega}|f(x)|
$$

where

$$
m_{1}=\sup _{M}\left|\left(-c_{n} \Delta+R_{0}\right)\left(\chi \varphi_{0}+1-\chi\right)\right| .
$$

Thus, if we want $\mathcal{L}(\bar{u}) \geq 0$ on $M \backslash \Omega$, we have to assume that

$$
-m_{1}+\delta^{N-1} m_{0}^{N} \inf _{x \in M \backslash \Omega}|f(x)| \geq 0
$$

that's

$$
\delta^{N-1} \inf _{x \in M \backslash \Omega}|f(x)| \geq m_{1} m_{0}^{-N} .
$$

It is clear that the existence of $\delta>0$ satisfying both (3.6) and (3.8) is equivalent to condition (H2) with $C_{\Omega}=\frac{\lambda_{D} m_{0}^{N}}{m_{1}}$. This achieves the proof of Proposition 3.1. 
Proposition 3.1 allows us to prove uniform $L^{\infty}$-estimates on the flow.

Proposition 3.2. Let $0<u_{0} \in C^{\infty}(M)$ such that $u_{0} \leq \bar{u}$ where $\bar{u}$ is given by Proposition 3.1. Then the solution $u$ of (1.4) satisfies for any $(t, x) \in[0,+\infty) \times M$

$$
\min \left(C_{0}, \min _{M} u_{0}\right) \leq u(t, x) \leq \max _{M} \bar{u}
$$

where $C_{0}=\left(\frac{\min _{M}\left|R_{0}\right|}{\max _{M}|f|}\right)^{\frac{n-2}{4}}$

Proof. First observe that the first inequality in (3.9) is already proved in Proposition 2.1. It remains then to prove the second inequality, that's,

$$
u(t, x) \leq \max _{M} \bar{u}
$$

Let $v=\bar{u}-u$. Since $u$ satisfies (1.4) and $\bar{u}$ satisfies (3.2), then we have

$$
\partial_{t}\left(\bar{u}^{N}-u^{N}\right) \geq \frac{n+2}{4}\left(c_{n} \Delta v-R_{0} v+f\left(\bar{u}^{N}-u^{N}\right)\right) .
$$

We have $\bar{u}^{N}-u^{N}=a v$, where

$$
a(t, x)=N \int_{0}^{1}(s \bar{u}(t, x)+(1-s) u(t, x))^{N-1} d s,
$$

so it follows from (3.10) that

$$
\partial_{t}(a v) \geq \frac{n+2}{4}\left(c_{n} \Delta v-R_{0} v+a f v\right) .
$$

Since $v(0, x)=\bar{u}(x)-u_{0}(x) \geq 0$, then by applying the maximum principle to (3.11) we get $v(t, x) \geq 0$ for any $t \geq 0$, that's

$$
u(t, x) \leq \bar{u}(x) .
$$

Proposition 3.2 is then proved.

Now we prove that the integral estimate (2.6) in Proposition 2.2 can be improved when $t \rightarrow+\infty$. More precisely, we have

Proposition 3.3. Let $0<u_{0} \in C^{\infty}(M)$ such that $u_{0} \leq \bar{u}$ where $\bar{u}$ is given by Proposition 3.1. Let $g(t)$ the solution of (1.3) given by Theorem 1.1 such that $g(0)=u_{0}^{\frac{4}{n-2}} g_{0}$. Then we have for any $p \geq 1$

$$
\lim _{t \rightarrow+\infty} \int_{M}\left|R_{g(t)}-f\right|^{p} d V_{g(t)}=0
$$


Proof. In what follows $C$ denotes a positive constant depending only on $u_{0}, g_{0}, f, p$, and its value may change from line to line.

We have by (2.2) for any $t \geq 0$,

$$
\frac{n-2}{2} \int_{0}^{t} \int_{M}\left|R_{g}-f\right|^{2} d V_{g}=\mathcal{E}(g(0))-\mathcal{E}(g(t)) .
$$

On the other hand, we have

$$
\mathcal{E}(g(t))=\int_{M}\left(c_{n}|\nabla u|^{2}+R_{0} u^{2}-\frac{n-2}{n} f u^{\frac{2 n}{n-2}}\right) d V_{g_{0}}
$$

and since $u$ is uniformly bounded by Proposition 3.2, then we have $\mathcal{E}(g(t)) \geq-C$. So it follows from (3.13) that

$$
\int_{0}^{+\infty} \int_{M}\left|R_{g(t)}-f\right|^{2} d V_{g(t)} \leq C .
$$

Since by Proposition 3.2 the volume of $g(t)$ is uniformly bounded, then it suffices to prove (3.12) for a sequence $p_{k} \rightarrow+\infty$. We shall prove (3.12) by induction when $p=p_{k}$, where

$$
p_{k}:=\frac{n}{2}\left(\frac{n}{n-2}\right)^{k}, k \in \mathbb{N}
$$

First we prove (3.12) for $p_{0}=\frac{n}{2}$. As in the proof of Proposition 2.2, if we use Lemma 2.1 and the fact that $u$ is uniformly bounded by Proposition 3.2, then one has for any $p>1$ :

$$
\begin{aligned}
\frac{d}{d t} \int_{M}\left|R_{g}-f\right|^{p} d V_{g}+ & C^{-1}\left(\int_{M}\left|R_{g}-f\right|^{\frac{p n}{n-2}} d V_{g}\right)^{\frac{n-2}{n}} \leq C \int_{M}\left|R_{g}-f\right|^{p} d V_{g} \\
& +\left(p-\frac{n}{2}\right) \int_{M}\left|R_{g}-f\right|^{p+1} d V_{g} .
\end{aligned}
$$

Set

$$
\phi_{p}(t)=\int_{M}\left|R_{g}-f\right|^{p} d V_{g} .
$$

If $p_{0}<2$, then by using Hölder's inequality and the fact that $u$ is uniformly bounded, we have

$$
\phi_{p_{0}} \leq C \phi_{2}^{\frac{p_{0}}{2}} \text {. }
$$

So it follows from (3.15) by taking $p=p_{0}=\frac{n}{2}$ that

$$
\frac{d}{d t} \phi_{p_{0}}^{\frac{2}{p_{0}}} \leq C \phi_{2} .
$$

By (3.14) there is a sequence $t_{\nu} \rightarrow+\infty$ such that $\phi_{2}\left(t_{\nu}\right) \rightarrow 0$ and $\int_{t_{\nu}}^{+\infty} \phi_{2}(s) d s \rightarrow 0$. So by integrating (3.17) on $\left[t_{\nu}, t\right]$ and using (3.16) we get

$$
\phi_{p_{0}}^{\frac{2}{p_{0}}}(t) \leq \phi_{p_{0}}^{\frac{2}{p_{0}}}\left(t_{\nu}\right)+C \int_{t_{\nu}}^{t} \phi_{2}(s) d s \leq C \phi_{2}\left(t_{\nu}\right)+C \int_{t_{\nu}}^{t} \phi_{2}(s) d s
$$

Letting $t \rightarrow+\infty$ and $\nu \rightarrow+\infty$ we obtain $\phi_{p_{0}}(t) \rightarrow 0$ as $t \rightarrow+\infty$. 
If $p_{0} \geq 2$, by using Hölder's inequality and Young's inequality we have for any $\varepsilon>0$,

$$
\int_{M}\left|R_{g}-f\right|^{p_{0}} d V_{g} \leq \varepsilon\left(\int_{M}\left|R_{g}-f\right|^{\frac{p_{0} n}{n-2}} d V_{g}\right)^{\frac{n-2}{n}}+\varepsilon^{-\frac{n\left(p_{0}-2\right)}{4}}\left(\int_{M}\left|R_{g}-f\right|^{2} d V_{g}\right)^{\frac{p_{0}}{2}} .
$$

By taking $\varepsilon=\frac{1}{2} C^{-1}$, where $C^{-1}$ is the constant appearing in (3.15), we obtain from (3.15)(where we take $p=p_{0}=\frac{n}{2}$ ),

$$
\frac{d}{d t} \phi_{p_{0}}+C^{-1} \phi_{\frac{p_{0} n}{n-2}}^{\frac{n-2}{n}} \leq C \phi_{2}^{\frac{p_{0}}{2}}
$$

But by Hölder's inequality, since the volume of $g$ is uniformly bounded, we have $\phi_{2} \leq C \phi_{p_{0}}^{\frac{2}{p_{0}}}$ and $\phi_{p_{0}} \leq C \phi_{\frac{p_{0}}{n-2}}^{\frac{n-2}{n}}$. Thus it follows from (3.18) that

$$
\frac{d}{d t} \phi_{p_{0}}^{\frac{2}{p_{0}}}+C^{-1} \phi_{p_{0}}^{\frac{2}{p_{0}}} \leq C \phi_{2}
$$

If we integrate (3.19) on $[0, t]$ and using (3.14) we get

$$
\int_{0}^{t} \phi_{p_{0}}^{\frac{2}{p_{0}}}(s) d s \leq C
$$

which implies, since $t \geq 0$ is arbitrary,

$$
\int_{0}^{+\infty} \phi_{p_{0}}^{\frac{2}{p_{0}}}(s) d s \leq C
$$

Thus there exists a sequence $t_{\nu} \rightarrow+\infty$ such that $\phi_{p_{0}}^{\frac{2}{p_{0}}}\left(t_{\nu}\right) \rightarrow 0$ as $\nu \rightarrow+\infty$. If we intgerate again (3.19) on $\left[t_{\nu}, t\right]$, we obtain

$$
\phi_{p_{0}}^{\frac{2}{p_{0}}}(t) \leq \phi_{p_{0}}^{\frac{2}{p_{0}}}\left(t_{\nu}\right)+C \int_{t_{\nu}}^{t} \phi_{2}(s) d s
$$

By using (3.14), it follow that $\phi_{p_{0}}^{\frac{2}{p_{0}}}(t) \rightarrow 0$ as $t \rightarrow+\infty$.

Now suppose by induction that

$$
\lim _{t \rightarrow+\infty} \phi_{p_{k}}(t)=0
$$

First let us prove that

$$
\lim _{t \rightarrow+\infty} \int_{t}^{t+1} \phi_{p_{k+1}^{n}}^{\frac{n-2}{n}}(s) d s=0 .
$$

We may suppose $k \geq 1$. Indeed, if $k=0$ (that's $p_{k}=p_{0}=\frac{n}{2}$ ), then (3.21) follows directly from (3.15) (with $p=\frac{n}{2}$ ) by integrating on $[t, t+1]$ and using (3.20). Thus let us prove (3.21) when $k \geq 1$.

By using Hölder's inequality and Young's inequality we have for any $p>\frac{n}{2}$ and $\varepsilon>0$,

$$
\int_{M}\left|R_{g}-f\right|^{p+1} d V_{g} \leq \varepsilon\left(\int_{M}\left|R_{g}-f\right|^{\frac{p n}{n-2}} d V_{g}\right)^{\frac{n-2}{n}}+\varepsilon^{-\frac{n}{2 p-n}}\left(\int_{M}\left|R_{g}-f\right|^{p} d V_{g}\right)^{1+\frac{2}{2 p-n}}
$$


By taking $p=p_{k}, \varepsilon=\frac{1}{2} C^{-1}$, where $C$ is the constant appearing in (3.15), we obtain from (3.15)

$$
\frac{d}{d t} \phi_{p_{k}}+\frac{1}{2} C^{-1} \phi_{p_{k+1}^{n}}^{\frac{n-2}{n}} \leq C \phi_{p_{k}}^{1+\frac{2}{2 p_{k}-n}}+C \phi_{p_{k}}
$$

Then (3.21) follows by integrating on $[t, t+1]$ and using (3.20).

Now if we apply (3.22) by taking $p=p_{k+1}$ and $\varepsilon=\frac{C^{-1}}{p_{k+1}-\frac{n}{2}}$, where $C$ is the constant appearing in (3.15), we obtain from (3.15) (where we take $p=p_{k+1}$ )

$$
\frac{d}{d t} \phi_{p_{k+1}} \leq C \phi_{p_{k+1}}^{1+\alpha_{k}}+C \phi_{p_{k+1}}
$$

where $\alpha_{k}=\frac{2}{2 p_{k+1}-n}$. The last inequality is equivalent to

$$
\frac{d}{d t} \log \phi_{p_{k+1}} \leq C\left(\phi_{p_{k+1}}^{\alpha_{k}}+1\right) .
$$

By (3.21) there is a sequence $t_{\nu} \rightarrow+\infty$ such that $\nu \leq t_{\nu} \leq \nu+1$ satisfying $\phi_{p_{k+1}}\left(t_{\nu}\right) \rightarrow 0$ as $\nu \rightarrow+\infty$. If we integrate (3.23) on $\left[t_{\nu}, t\right]$ where $t \in[\nu, \nu+1]$, we obtain

$$
\log \frac{\phi_{p_{k+1}}(t)}{\phi_{p_{k+1}}\left(t_{\nu}\right)} \leq C\left(\int_{\nu}^{\nu+1} \phi_{p_{k+1}}^{\alpha_{k}}(s) d s+1\right) .
$$

We note here that $\alpha_{k} \leq \frac{n-2}{n}$, so by Hölder's inequality we have

$$
\int_{\nu}^{\nu+1} \phi_{p_{k+1}}^{\alpha_{k}}(s) d s \leq\left(\int_{\nu}^{\nu+1} \phi_{p_{k+1}^{n}}^{\frac{n-2}{n}}(s) d s\right)^{\frac{n \alpha_{k}}{n-2}} \rightarrow 0 \text { as } \nu \rightarrow+\infty
$$

by (3.21). Thus it follows from (3.24)

$$
\log \frac{\phi_{p_{k+1}}(t)}{\phi_{p_{k+1}}\left(t_{\nu}\right)} \leq C
$$

which implies that $\phi_{p_{k+1}}(t) \rightarrow 0$ as $t \rightarrow+\infty$. The proof of Proposition 3.3 is then complete.

Now we can prove uniform $C^{\alpha}$-estimates on the solution.

Proposition 3.4. Let $0<u_{0} \in C^{\infty}(M)$ such that $u_{0} \leq \bar{u}$ where $\bar{u}$ is given by Proposition 3.1. Then the solution $u$ of (1.4) satisfies for some $\alpha \in(0,1)$

$$
\|u\|_{C^{\alpha}([0,+\infty) \times M)} \leq C,
$$

where $C$ is a positive constant depending only on $u_{0}, g_{0}$ and $f$.

Proof. By using Proposition 3.2 and Proposition 3.3, the proof is identical to that of Proposition 2.6 in Brendle [6].

Now we are in position to prove Theorem 1.2. 
Proof of Theorem 1.2. Let $g=u^{\frac{4}{n-2}} g_{0}$ the solution of (1.3) given by Theorem 1.1. By Proposition 3.2 we have that $u$ is bounded from below and above uniformly on $[0,+\infty)$. As in the proof of Theorem 1.1, this implies that equation (1.4) is uniformly parabolic and by Proposition 3.4 we have uniform $C^{\alpha}$-bound on the solution $u$ on $[0,+\infty) \times M$. We then apply the classical regularity theory of linear parabolic equations to obtain uniform $C^{k}$-bound for any $k \in \mathbb{N}$, that's

$$
\|u(t)\|_{C^{k}(M)} \leq C_{k}
$$

for some constant $C_{k}$ independent of $t$. It follows from (3.25) that there is a sequence $t_{\nu} \rightarrow+\infty$ such that $u\left(t_{\nu}\right)$ converges in $C^{k}(M)$ for any $k \in \mathbb{N}$, to some function $u_{\infty} \in C^{\infty}(M)$. Since $u(t)$ is uniformly bounded from below by Proposition 3.2, then we have $u_{\infty}>0$. By using Proposition 3.3 and Passing to the limit when $\nu \rightarrow \infty$, we see that $R_{g_{\infty}}=f$, where $g_{\infty}=u_{\infty}^{\frac{4}{n-2}} g_{0}$, that's $f$ is the scalar curvature of $g_{\infty}$. By the general result of Simon [19] on evolution equations, $u_{\infty}$ is the unique limit of $u(t)$ when $t \rightarrow+\infty$.

Proof of Corollary 1.1. Since $f<0$ almost everywhere on $M$, then for $\varepsilon>0$ small enough, the open set

$$
\Omega_{\varepsilon}=\{x \in M: f(x)>-\varepsilon\}
$$

has arbitrary small volume. This implies that the first eigenvalue $\mu_{\Omega_{\varepsilon}}$ of $-c_{n} \Delta$ on $\Omega_{\varepsilon}$ with zero Dirichlet conditions is arbitrary large if $\varepsilon$ is small enough. But since

$$
\lambda_{\Omega_{\varepsilon}} \geq \mu_{\Omega_{\varepsilon}}+\min _{M} R_{0}
$$

then we have $\lambda_{\Omega_{\varepsilon}}>0$ if $\varepsilon$ is small enough. Thus the condition (H1) is satisfied with $\Omega=\Omega_{\varepsilon}$. Condition (H2) is also satisfied since by continuity of $f$ we have $f \leq 0$ everywhere on $M$.

Proof of Theorem 1.3. Suppose that condition (H1) is not satisfied, that's, for any open set $\Omega \subset M$ such that $f<0$ on $M \backslash \Omega$, we suppose $\lambda_{\Omega} \leq 0$. For $\varepsilon>0$, consider the following familly of open sets :

$$
\Omega_{\varepsilon}=\{x \in M: f(x)>-\varepsilon\} .
$$

For the simplicity of notation we set $\lambda_{\varepsilon}=\lambda_{\Omega_{\varepsilon}}$. According to our hypothesis we have then

$$
\lambda_{\varepsilon} \leq 0 \text { for all } \varepsilon>0 .
$$

By using Sard's theorem, there exists a sequence $\varepsilon_{n} \rightarrow 0$ such that $\varepsilon_{n}$ is a regular value of $f$ and then $\Omega_{\varepsilon_{n}}$ has a smooth boundary $\partial \Omega_{\varepsilon_{n}}=\left\{x \in M: f(x)=-\varepsilon_{n}\right\}$.

Let $\varphi_{n}$ an eigenfunction of $-c_{n} \Delta+R_{0}$ associated with $\lambda_{\varepsilon_{n}}$. As already mentioned in the proof of proposition 3.2, we have by the maximum principle that

$$
\varphi_{n}>0 \text { on } \Omega_{\varepsilon_{n}} \text { and } \frac{\partial \varphi_{n}}{\partial \nu} \leq 0 \text { on } \partial \Omega_{\varepsilon_{n}}
$$

where $\nu$ is the outer normal vector to $\partial \Omega_{\varepsilon_{n}}$. By normalising if necessary, we may assume that

$$
\int_{\Omega_{\varepsilon_{n}}} \varphi_{n} d V_{g_{0}}=1
$$


If we multiply equation (1.4) by $\varphi_{n}$ and integrate on $\Omega_{\varepsilon_{n}}$, we have

$$
\frac{d}{d t} \int_{\Omega_{\varepsilon_{n}}} u^{N} \varphi_{n} d V_{g_{0}}=\frac{n+2}{4} \int_{\Omega_{\varepsilon_{n}}}\left(c_{n} \Delta u-R_{0} u\right) \varphi_{n} d V_{g_{0}}+\frac{n+2}{4} \int_{\Omega_{\varepsilon_{n}}} f u^{N} \varphi_{n} d V_{g_{0}} .
$$

An integration by parts gives

$$
\int_{\Omega_{\varepsilon_{n}}}\left(c_{n} \Delta u-R_{0} u\right) \varphi_{n} d V_{g_{0}}=-\lambda_{\varepsilon_{n}} \int_{\Omega_{\varepsilon_{n}}} u \varphi_{n} d V_{g_{0}}-c_{n} \int_{\partial \Omega_{\varepsilon_{n}}} \frac{\partial \varphi_{n}}{\partial \nu} u d V_{g_{0}} .
$$

Since $\lambda_{\varepsilon_{n}} \leq 0$, then we obtain by using (3.27)

$$
\int_{\Omega_{\varepsilon_{n}}}\left(c_{n} \Delta u-R_{0} u\right) \varphi_{n} d V_{g_{0}} \geq-\lambda_{\varepsilon_{n}} \inf _{M} u-c_{n} \inf _{M} u \int_{\partial \Omega_{\varepsilon_{n}}} \frac{\partial \varphi_{n}}{\partial \nu} d V_{g_{0}}
$$

On the other hand we have

$$
c_{n} \int_{\partial \Omega_{\varepsilon_{n}}} \frac{\partial \varphi_{n}}{\partial \nu} d V_{g_{0}}=c_{n} \int_{\Omega_{\varepsilon_{n}}} \Delta \varphi_{n} d V_{g_{0}}=\int_{\Omega_{\varepsilon_{n}}}\left(-\lambda_{\varepsilon_{n}}+R_{0}\right) \varphi_{n} d V_{g_{0}}
$$

and by using (3.28) we get

$$
-c_{n} \int_{\partial \Omega_{\varepsilon_{n}}} \frac{\partial \varphi_{n}}{\partial \nu} d V_{g_{0}} \geq \lambda_{\varepsilon_{n}}+\inf _{M} R_{0}
$$

Combining (3.30) and (3.31) we obtain

If we substitute in (3.29) we get

$$
\int_{\Omega_{\varepsilon_{n}}}\left(c_{n} \Delta u-R_{0} u\right) \varphi_{n} d V_{g_{0}} \geq \inf _{M} R_{0} \inf _{M} u
$$

$$
\frac{d}{d t} \int_{\Omega_{\varepsilon_{n}}} u^{N} \varphi_{n} d V_{g_{0}} \geq \frac{n+2}{4} \inf _{M} R_{0} \inf _{M} u+\frac{n+2}{4} \int_{\Omega_{\varepsilon_{n}}} f u^{N} \varphi_{n} d V_{g_{0}} .
$$

By Proposition 3.2 we have $u \geq C_{0}$, where $C_{0}$ is a positive constant depending only on $u_{0}, g_{0}$ and $f$. It follows from (3.32) by using the fact that $f>-\varepsilon_{n}$ on $\Omega_{\varepsilon_{n}}$,

$$
\frac{d}{d t} \int_{\Omega_{\varepsilon_{n}}} u^{N} \varphi_{n} d V_{g_{0}} \geq C-\frac{n+2}{4} \varepsilon_{n} \int_{\Omega_{\varepsilon_{n}}} u^{N} \varphi_{n} d V_{g_{0}},
$$

where $C$ is a positive constant depending only on $u_{0}, g_{0}$ and $f$. By integrating this differential inequality on $[0, t]$, we get

$$
\int_{\Omega_{\varepsilon_{n}}} u^{N}(t) \varphi_{n} d V_{g_{0}} \geq \int_{\Omega_{\varepsilon_{n}}} u_{0}^{N} \varphi_{n} d V_{g_{0}}+C t-\frac{n+2}{4} \varepsilon_{n} \int_{0}^{t} \int_{\Omega_{\varepsilon_{n}}} u^{N}(s) \varphi_{n} d V_{g_{0}} d s,
$$

which implies by using (3.28)

$$
\max _{x \in M} u^{N}(t, x) \geq C t-\frac{n+2}{4} \varepsilon_{n} \int_{0}^{t} \max _{x \in M} u^{N}(s, x) d s .
$$

Letting $n \rightarrow+\infty$, we obtain

$$
\max _{x \in M} u^{N}(t, x) \geq C t
$$

The proof of Theorem 1.3 is complete. 


\section{REFERENCES}

[1] A. Ambrosetti, A. Malchiodi, A multiplicity result for the Yamabe problem on $\mathbb{S}^{n}$, J. Funct. Anal. 168 (1999), 529-561.

[2] T. Aubin, Equations différentielles non linéaires et problème de Yamabe concernant la courbure scalaire, J. Math. Pures Appl. 55 (1976), 269-296.

[3] P. Baird, A. Fardoun, R.Regbaoui, The evolution of the scalar curvature of a surface to a prescribed function, Ann. Sc. Norm. Super. Pisa. Cl. Sci. (5) 3 (2004), 17-38.

[4] A. Besse, Einstein manifolds, Springer, Berlin (1976).

[5] S. Bismuth, Prescribed scalar curvature on a complete Riemannian manifold in the negative case, J. Math. Pures Appl. 79 (2000), 941-951.

[6] S. Brendle, Convergence of the Yamabe flow for arbitrary initial energy, J. Differ. Geom 69 (2005), 217-278.

[7] S. Brendle, Convergence of the Yamabe flow in dimension 6 and higher, Invent. Math. 170 (2007), 541-576.

[8] B. Chow, The Yamabe flow on locally conformally flat manifolds with positive Ricci curvature, Comm. Pure Appl. Math. 45 (1992), 1003-1014.

[9] X. Chen, X. Xu, The scalar curvature flow on $\mathbb{S}^{n}$-perturbation theorem revisited, Invent. Math. 86 (1986), 243254.

[10] J. Escobar, R. Schoen, Conformal metrics with prescribed scalar curvature, Invent. Math. 187 (2012), 395-506.

[11] R. Hamilton, The Ricci flow on surfaces, Mathematics and General Relativity, Comtemporary Math. 187, Amer. Math. Soc., Provdence, RI (1988), 237-262.

[12] E. Hebey, M. Vaugon, Le problème de Yamabe équivariant, Bull. Sci. Math. 117 (1993), 241-286.

[13] J. Kazdan, F. Warner, Existence and conformal deformation of metrics with prescribed Gaussian and scalar curvatures, Ann. of Math. 101 (1975), 317-331.

[14] J-M. Lee, M. Parker, The Yamabe problem, Bull. Am. Math. Soc. 17 (1987), 37-91.

[15] A. Rauzy, Courbures scalaires des varits d'invariant conforme ngatif, Trans. Amer. Math. Soc. 347 (1995), .

[16] R. Schoen, Conformal deformation of a Riemanniannmetric to constant scalar curvature, J. Differ. Geom. 20 (1984), 4729-4745.

[17] R. Schoen, A report on some recent progress on nonlinear problems in geometry, In: Surveys in Differential Geometry, Lehigh University, Bethlehem, PA (1991), $201 ? 241$.

[18] H. Schwetlick, M. Struwe, Convergence of the Yamabe flowfor large energies, J. Reine. Angew. Math. 562 (2003), 59-100.

[19] L. Simon, Asymptotics for a class of non-linear evolution equations with applications to geometric problems,, Ann. of Math. 118 (1983), 525-571.

[20] M. Struwe, A flow approach to Nirenberg's problem, Duke Math. J. 128 (2005), 19-64.

[21] J. Vazquez, L. Véron, Solutions positives d'quations elliptiques semi-linaires sur des varits riemanniennes compactes, C. R. Acad. Sei. Paris. Sér. I Math. 312 (1991), 811-815.

[22] R. Ye, Global existence and convergence of the Yamabe flow, J. Differ. Geom. 39 (1994), 35-50. 
Laboratoire de Mathématiques, UMR 6205 CNRS Université de Bretagne Occidentale 6 Avenue Le Gorgeu, 29238 Brest Cedex 3 France

E-mail address: Inas. Amacha@univ-brest.fr

Laboratoire de Mathématiques, UMR 6205 CNRS Université de Bretagne Occidentale 6 Avenue Le Gorgeu, 29238 Brest Cedex 3 France

E-mail address: Rachid.Regbaoui@univ-brest.fr 